\title{
Electron Diffraction Study of Rhenium Fluorides. I. Heavy-Atom Corrections and Structure of $\operatorname{ReF}_{6}{ }^{*}$
}

\author{
E. Jean Jacob and L. S. Bartelit $\dagger$ \\ Department of Chemistry, University of Michigan, Ann Arbor, Michigan 48104
}

(Received 13 May 1970)

\begin{abstract}
To date, no theoretical models for electron molecular scattering have been developed which bring calculated scattered intensities from heavy atoms into satisfactory agreement with experiment. Preparatory to an electron diffraction study of $\mathrm{ReF}_{7}$, a molecule with an uncertain and possibly complex structure, an investigation of $\mathrm{ReF}_{6}$ was undertaken. The simplicity of the hexafluoride's geometry ( $O_{h}$ symmetry) allows an empirical characterization of the discrepancy between experimental and calculated intensities, a discrepancy which can be attributed chiefly to the behavior of ReF interference terms rather than to uncertainties in structural parameters. After a structure analysis of $\operatorname{ReF}_{6}$, the observed difference signal ( $I^{\text {obs }}-I^{\text {calc }}$ ) was used with appropriate scaling to modify $\mathrm{ReF}_{7}$ intensities before structural parameters for the heptafluoride were refined. Parameters determined for $\mathrm{ReF}_{6}$, e.g., $r_{g}(\operatorname{ReF})=1.832 \pm 0.004 \AA$ and $l_{o}(\mathrm{ReF})=0.044 \AA$, are in excellent agreement with those reported from diffraction studies of other $5 d$ transition-metal hexafluorides. Results for $\mathrm{ReF}_{7}$ are reported in Paper II.
\end{abstract}

\section{INTRODUCTION}

At the present imperfect stage of development of electron scattering theory for molecules containing very heavy atoms, there is a serious question about the reliability of structural parameters that can be derived for a molecule such as rhenium heptafluoride from a conventional electron diffraction analysis. Recent diffraction studies of several tungsten, osmium, iridium, and platinum compounds, ${ }^{1-4}$ have clearly demonstrated that the discrepancies between calculated and experimental curves for these molecules, while relatively large, are highly characteristic in nature and (qualitatively) transferrable from molecule to molecule. As long as a molecule has a high symmetry, e.g., tetrahedral or octahedral, with well-resolved peaks in the radial distribution curves, these misfits probably do not severely bias the determination of structural parameters. Furthermore, comparisons among heavy-atom molecules of this type should be roughly as reliable as comparisons among lighter molecules, owing to the consistency in shape and magnitude of the differences between calculated and experimental curves for molecules with bonds of $\operatorname{similar} Z_{i}$ and $Z_{j}$. This provides the hope that structure determinations for molecules of low or unknown symmetry may be improved empirically by the information derived from the study of related, but highly symmetrical molecules.

Both for $\mathrm{IF}_{7}$ and $\mathrm{ReF}_{7}$ the observed vibrational spectra suggest a $D_{5 h}$ symmetry. ${ }^{5}$ However, a recent electron diffraction study of $\mathrm{IF}_{7}{ }^{6}$ has revealed the presence of significant distortions of the thermal average structure from $D_{5 h}$. Differences in the spectra of the two compounds are striking enough ${ }^{5}$ to make $\mathrm{ReF}_{7}$ a candidate for even greater departures from $D_{5 h}$ than were found for $\mathrm{IF}_{7}$. It is of additional interest to discover whether the heptafluorides are compatible with a simple point-repulsion model proposed to account for the structure of $\mathrm{MX}_{r}$ molecules. ${ }^{7}$ Therefore, we undertook an electron diffraction study of $\mathrm{ReF}_{7}$.
In order to maximize the amount of structural information derivable from the electron diffraction data, within the framework of present scattering theory, we felt it essential that some attempt be made to remove the bias introduced by the nonrandom differences between theory and experiment. Therefore, we chose to use rhenium hexafluoride to "calibrate" the misfit expected for $\mathrm{ReF}$ molecular scattering. The suitability of $\mathrm{ReF}_{6}$ as a standard appeared plausible though not unequivocal. It was necessary to look at it for its own sake in view of its dynamic Jahn-Teller behavior ${ }^{8-10}$ stemming from its degenerate electronic ground state.

\section{EXPERIMENTAL PROCEDURE}

Samples of $\operatorname{ReF}_{6}$ and $\mathrm{ReF}_{7}$ in Monel vessels with stainless-steel fittings were provided by Argonne National Laboratory and were used without further purification. All experimental procedures for collecting and processing the diffraction data were identical for the two compounds.

Patterns for scattering by $40-\mathrm{kV}$ incident electrons were recorded on $4 \times 5$ in. Kodak process plates at 21 - and $11-\mathrm{cm}$ camera distances, using a rotating $R^{3}$ sector of $4.8-\mathrm{cm}$ radius. The diffraction apparatus was that constructed at Iowa State University ${ }^{11}$ but presently in operation at the University of Michigan.

All surfaces of the inlet system to the diffraction chamber with which the sample came into contact were either nickel or Monel and were preseasoned with an active fluorinating agent, $\mathrm{XeF}_{6}$, after each exposure to the atmosphere and before introduction of the sample. The samples were introduced into the diffraction chamber by cooling the sample vessel to a temperature corresponding to a convenient vapor pressure (10-50 torr), expanding the sample into an evacuated section of the inlet line, closing the sample vessel, then letting the vapor out the nozzle in a burst to interact with the electron beam. Beam currents of $0.4-0.5 \mu \mathrm{A}$ and exposure times of 5-6 sec yielded absorbances in the range $0.3-0.9$. 
Tabif I. Molecular parameters for rhenium hexafluoride."

\begin{tabular}{|c|c|c|c|c|c|c|c|}
\hline & & $r_{f}(\mathrm{ReF})$ & $l_{g}(\mathrm{ReF})$ & $l_{g}\left(\mathrm{~F}+\mathrm{H}^{\prime}\right)$ & $l_{g}(\mathrm{~F} \cdots \mathrm{F})$ & $\begin{array}{c}\text { Experimental } \\
\text { shrinkages } \\
\delta(\mathrm{FF}) / \delta(\mathrm{F} \cdots \mathrm{F})\end{array}$ & $\sigma\left(I_{0}\right) /\left\langle I_{0}\right\rangle_{\mathrm{Av}}$ \\
\hline$A$ & $\begin{array}{l}\text { Uniform weighting of } I_{0}(s) \text {; } \\
\text { unconstrained model }\end{array}$ & 1.8265 & 0.0437 & 0.0916 & 0.0467 & $-0.0003 / 0.002$ & 0.0029 .3 \\
\hline IB & $\begin{array}{l}\text { Uniform weighting of } s I_{0}(s) \text {; } \\
\text { unconstrained model }\end{array}$ & 1.8318 & 0.0440 & 0.1004 & 0.0600 & $0.005 / 0.012$ & 0.00124 \\
\hline C & $\begin{array}{l}\text { Uniform weighting of } s I_{0}(s) \\
\mathrm{O}_{h} \text { model; single scale factor }\end{array}$ & 1.8316 & 0.0440 & 0.1000 & 0.0600 & $\cdots$ & 0.00125 \\
\hline $\mathrm{D}$ & $\begin{array}{l}\text { Uniform weighting of } s I_{0}(s) \\
O_{h} \text { model; FF scale factor } \\
\text { augmented by } 25 \%\end{array}$ & 1.8319 & 0.0431 & 0.1157 & 0.0718 & $\cdots$ & 0.00116 \\
\hline $\mathrm{E}$ & $\sigma(\theta)$ neglecting systematic errors ${ }^{e}$ & 0.00065 & 0.00073 & 0.0030 & 0.0066 & & \\
\hline F & $\sigma(\theta)$ from "noise" in $f(r) d$ & 0.0045 & 0.0045 & 0.0027 & $\cdots$ & & \\
\hline G & $\begin{array}{l}\text { Net } \sigma(\theta) \text {, including experimen } 1 \text { al } \\
\text { uncertaintiese }\end{array}$ & 0.0047 & 0.0046 & $0.0036^{f}$ & 0.0067 & & \\
\hline
\end{tabular}

a Based on least-squares fits to 109 blended intensity points at intervals of $\Delta s=\pi / 10$. Units are angstroms, except for $\sigma^{+} \mathrm{s}$.

b Spectroscopic values for $\delta(\mathrm{FF})$ and $\delta(\mathrm{F} \ldots \mathrm{F})(0.0009$ and $0.0050 \AA$, respectively) were used in the refinement of $\mathrm{O}_{h}$ models. Uncertainties in $r_{F F}$ and $\gamma_{F} \ldots \mathbf{F}$ are estimated to be 0.0036 and $0.0073 \AA$ for analysis $B$.

${ }^{c}$ Conventional least-squares $\sigma(\theta)$ 's for analysis B (see footnote $b$ of Table II) adjusted for correlations between intensity points, using an intensity correlation parameter, $\gamma=1 \AA$. See L. S. Bartell, in Physical Methods in Chemisiry, edited by A. Weissberger and B. W. Rossiter (Interscience, New York, to be published), 4th ed., Electron Diffraction chavter.

dUses "noise" in the region of the peak corresponding to $r_{i j}$, together with the peak height and effective amplitude, to estimate $\sigma\left(r_{i j}\right)$ and $\sigma\left(l_{i j}\right)$. See reference of Footnote $c$ above.

Five or six plates at each camera distance were photometered using a modification of the fully automated digital mode of recording previously described. ${ }^{12}$ Absorbances were converted to exposures according to the expression $E=A+0.05 A^{3}$, where $A$ is the absorbance for a given reading and $E$ the corresponding exposure.

\section{PRELIMINARY TREATMENT OF DATA}

Experimental leveled intensities, $I_{0}(s)$, were derived from the exposures in a conventional manner. Experimental $s, I_{0}(s)$, and background functions, $I_{B}(s)$, determined in the course of the analysis for $\mathrm{ReF}_{6}$ and $\mathrm{ReF}_{7}$, respectively, are available from ASIS. ${ }^{13}$

Inelastic scattering factors of Heisenberg and Bewilogua ${ }^{14}$ were used for both rhenium and fluorine. Elastic scattered amplitudes for fluorine were taken from the partial wave calculations of Bonham and Cox, ${ }^{15}$ while those for rhenium were based on similar calculations performed recently in Bonham's laboratory. ${ }^{16,17} \mathrm{We}$ found that the rhenium and fluorine phase shifts corresponding to the scattered amplitudes adopted implied too large an $s_{c}$, the value of $s$ at which $\Delta \eta(s)$ for the bond equals $\pi / 2$. Experimental determi-
- Assumed experimental uncertainties of 2, 3, 4, and 4 parts jer 10000 . respectively, for errors in $r_{i j}$ and $l_{i j}$ arising from uncertainties in electron wavelength, camera distance, radial coordinate in microphotometer seanning, and from magnetic disturbance by sector mount were used. Frrots for amplitudes also include a factor to account for the strong correlations with the index of resolution, which factor was of the form $\sigma\left(l_{i j}\right)=0.7 \times$ $l_{i j} \times \sigma(R)$, where $\sigma(R)$ was assumed to be 0.03 . Rows $\mathrm{E}$ and $F$ are nutually exclusive estimates of goodness of fit. In each case, the larger of the two values was used in calculating the entry for row $G$.

${ }^{f}$ If $\sigma(R)$ for FF distances is taken to be 0.25 , say, $\sigma\left(l_{\mathrm{FF}}\right)$ would be $0.017 \AA$. While this is probably an overestimate, it does correspond closely to the observed shift in $l_{\mathrm{FF}}$ witl a $25 \%$ increase in the FF scale factor.

nations of $s_{c}$ with an uncertainty of $\pm 0.3 \AA^{-1}$ are possible, and such values, along with theoretical values calculated using the complex scattering factors of Ibers and Hoerni ${ }^{18}$ have been reported for several hexafluorides ${ }^{1,19}$ close to $\operatorname{ReF}_{6}$ in atomic number of the central atom, namely $\mathrm{WF}_{6}(13.7,13.8), \mathrm{OsF}_{6}(13.4$, 13.6), and $\operatorname{IrF}_{6}(13.3,13.5)$. By comparison, the $s_{c}$ of 14.6 $\AA^{-1}$ for the ReF bonds based on Bonham's scattering factors is significantly high. This is also borne out by comparison of our experimental reduced intensity curves with calculated ones in the region of $s_{c}$. Bonham-Ukaji phase shifts ${ }^{20}$ imply an $s_{c}$ of 13.6 for ReF bonds, in fair agreement with the present experiment." They were adopted without further attempt at optimization and used throughout the analysis of the data.

\section{PROCEDURES FOR REFINEMENT OF MOLECULAR PARAMETERS}

\section{Rhenium Hexafluoride}

Determination of molecular parameters for $\mathrm{ReF}_{6}$ was effected exclusively by least-squares refinements of experimental intensities for the individual camerat distances and for a composite of the intensities for the two data ranges. A variety of weighting functions was 
TABLE II. Correlation coefficients for rhenium hexafluoride. ${ }^{n}$

\begin{tabular}{|c|c|c|c|c|c|c|c|}
\hline $\begin{array}{c}\text { Parameter, } \\
\theta_{i}(\AA) \\
\left.\sigma^{0}\left(\theta_{i}\right)(\AA) \mathrm{Q}\right)\end{array}$ & $\begin{array}{c}r_{g}(\mathrm{ReF}) \\
0.00053\end{array}$ & $\begin{array}{r}r_{g}(\mathrm{lF}) \\
0.0035\end{array}$ & $\begin{array}{c}r_{g}(F \ldots F) \\
0.0104\end{array}$ & $\begin{array}{r}l_{g}(\mathrm{ReF}) \\
0.00060\end{array}$ & $\begin{array}{l}l_{g}(\mathrm{FF}) \\
0.0033\end{array}$ & $\begin{array}{r}l_{y}(\mathrm{~F} \cdots \mathrm{F}) \\
0.0098\end{array}$ & $\begin{array}{l}R^{\mathrm{c}} \\
0.017\end{array}$ \\
\hline$r_{g}(\mathrm{ReF})$ & 1 & -0.05 & 0.0 & 0.01 & 0.01 & -0.01 & 0.04 \\
\hline$r_{g}(\mathrm{FF})$ & & 1 & -0.01 & -0.11 & -0.10 & 0.0 & -0.15 \\
\hline$r_{G}(\mathrm{~F} \cdots \mathrm{F})$ & & & 1 & 0.0 & -0.02 & -0.02 & -0.01 \\
\hline$l_{g}(\mathrm{ReF})$ & & & & 1 & 0.32 & 0.07 & 0.72 \\
\hline$l_{g}(\mathbf{F F})$ & & & & & 1 & 00 it & 0.45 \\
\hline$l_{p}(\mathrm{~F} \cdots \mathrm{F})$ & & & & & & 1 & 0.09 \\
\hline$R$ & & & & & & & 1 \\
\hline
\end{tabular}

a From an unconstrained model as reported in row $B$ of Table $I$. The coefficients are evaluated directly from the $B^{-1}$ matrix according to the expression $\rho_{i j}=B_{i j}{ }^{-1} /\left(B_{i i}{ }^{-1} \times B_{j j^{-1}}\right)^{1 / 2}$. See W. C. Hamilton, Statistics in Physical Science (Ronald, New York, 1964), p. 127, for definition of the $B$ matrix.

used in the course of the analysis. Small shifts in bond length and vibrational amplitude result from weighting data at large values of $s$ relatively more heavily, as indicated by rows $a$ and $b$ of Table $I$. The shifts are consistent with those observed in other molecules containing heavy scatterers. ${ }^{23}$ Correlations among derived parameters are given in Table II.

Radial distribution curves were calculated using the $M_{n}(s)$ approximation ${ }^{22}$ for taking into account scattering from planetary electrons. Theoretical intensities from $s=0-5.6 \AA^{-1}$ were combined with modified experimental intensities from $s=5.3-39.3 \AA^{-1}$. A damping factor of $e^{-b s^{2}}$ with $b=0.0015 \AA^{2}$ was used. Asymmetry constants of $2.0 \AA^{-1}$ for the ReF and geminal $\mathrm{F} \cdots \mathrm{F}$ and $1.0 \AA^{-1}$ for the long $F \cdot \cdots F$ distances were assumed. No integral termination corrections were applied.

\section{Rhenium Heptafluoride}

After background functions were refined for the 21and $11-\mathrm{cm}$ camera ranges, a blended intensity function was constructed as described above, extending over the same $s$ range as the $\mathrm{ReF}_{6}$ intensities. This blended intensity was then modified on the basis of the $\mathrm{ReF}_{6}$ data in the following manner. The error signal for an $\mathrm{ReF}_{6}$ radial distribution curve $\left[f(\boldsymbol{r})_{\text {expt1 }}-f(\boldsymbol{r})_{\text {eule }}\right]$ from $r=0.75-3.75 \AA$ was first shifted by the difference

FIG. 1. Experimental and calculated reduced intensity functions for $\operatorname{ReF}_{6} ; \Delta s M(s)$ represents $\left[s M(s)_{\text {exptl }}-\right.$ $\left.s M(s)_{\text {cale }}\right]$. b Standard errors for parameters calculated assuming errors were statistically distributed. See, for example, Hamilton, teference of Footnote a above.

${ }^{\mathrm{C}}$ Index of resolution; dimensionless.

in mean bond length between $\mathrm{ReF}_{6}$ and $\mathrm{ReF}_{7}$ (approximately $0.01 \AA$ based on preliminary refinements of the $\mathrm{ReF}_{7}$ data), and scaled to account for the differences in indices of resolution $R$ and in molecular and atomic scattering for the two molecules according to the expression

$$
\text { scale }=R_{\operatorname{ReF}_{7}[}\left[I_{\text {at }}\left(\operatorname{ReF}_{6}\right) / 6 I_{\text {at }}\left(\operatorname{ReF}_{7}\right)\right]=1.17,
$$

where $I_{\mathrm{at}}=\sum_{i} Z_{i}\left(Z_{i}+1\right)$. A Fourier sine transform of the resultant modified $\Delta f(r)$ was then subtracted from the original experimental $s M(s)$ values for $\operatorname{ReF}_{7}$. All structure refinements for $\mathrm{ReF}_{7}$ reported in the following paper are based on these modified intensities or upon radial distribution curves constructed from them. The small shift in mean bond length and vibrational amplitude going from $\mathrm{ReF}_{6}$ to $\mathrm{ReF}_{7}$, and the relatively small spread among $\mathrm{ReF}$ bond lengths in $\mathrm{ReF}_{7}$ all enhance the likelihood that the above procedure, though admittedly crude, captures the essence of the "correction" to experimental intensities necessary to remove the bias which failures of scattering theory introduce.

\section{Structure of $\mathrm{ReF}_{6}$}

Figure 1 displays the experimental and calculated reduced intensity curves

$$
s M(s)=s\left\{\left[I_{0}(s) / I_{B}(s)\right]-1\right\}
$$

together with the corresponding difference curve. An experimental radial distribution curve

$$
f_{n}(r)=\int_{0}^{s(\max )} s M_{n}(s) \exp \left(-b s^{2}\right) \sin s r d s,
$$

with a difference curve for a typical model is shown in Fig. 2. Parameters derived in various least-squares intensity refinements are listed in Table I together with various estimates of the uncertainties in the parameters. Although it was found that an arbitrary increase of $25 \%$ in the scale factor for fluorine-fluorine distances relative to $\mathrm{ReF}$ distances gave roughly a $10 \%$ 


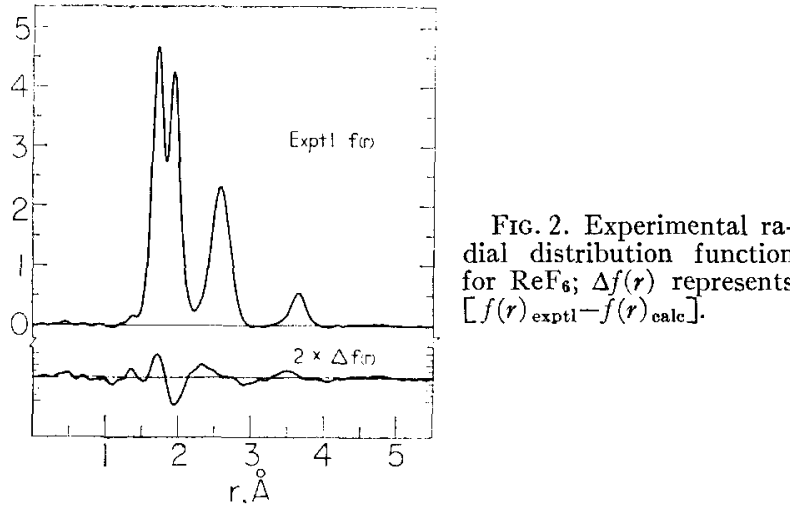

better fit to the data, ${ }^{24,25}$ the lack of any firm theoretical basis for such an assumption made us feel unjustified in using it for the correction to the $\mathrm{ReF}_{7}$ intensities.

We found that the electron diffraction data for rhenium hexafluoride contain no evidence for perturbation of structural parameters or mean amplitudes of vibration due to a dynamic Jahn-Teller effect despite the spectroscopic evidence for vibronic coupling in $\mathrm{ReF}_{6}{ }^{9}{ }^{9}$ To within the error limits of the present experiment, rhenium hexafluoride is a regular octahedron (see row c of Table I). Furthermore, the mean bond length determined for $\operatorname{ReF}_{6}\left(r_{g}=1.832 \AA\right)$ is consistent with bond lengths reported for the hexafluorides of several other $5 d$ transition metals, namely $W_{6} F_{6}{ }^{1,2}$ $\left(r_{g}=1.834 \AA ; r_{g}=1.832 \AA\right), \mathrm{OsF}_{6}{ }^{1} \quad\left(r_{\theta}=1.832 \AA\right)$, and $\operatorname{IrF}_{6}{ }^{1}\left(r_{g}=1.832 \AA\right)$. Although $\mathrm{OsF}_{6}$ is also a candidate for Jahn-Teller distortions, no deformation from $O_{h}$ symmetry was detected in the study by Schomaker et al. ${ }^{1}$

Inasmuch as there are no calculations of vibrational amplitudes for $\operatorname{ReF}_{6}$ based on a suitable vibronic wavefunction and energy levels, ${ }^{26}$ we must base our assessment of whether or not the diffraction experiment provides evidence for vibronically perturbed amplitudes on comparisons with experimental results for related, but presumably unperturbed molecules. Spectroscopic studies show that the vibrational frequencies of $\mathrm{WF}_{6}, \mathrm{ReF}_{6}, \mathrm{OsF}_{6}$, and $\mathrm{IrF}_{6}$ are remarkably similar, ${ }^{,, 27}$ suggesting that the series of molecules would have nearly identical vibrational amplitudes in the absence of vibronic interactions in $\mathrm{ReF}_{6}$ and $\mathrm{OsF}_{6}$. Amplitudes determined by electron diffraction are independent of any assumption about vibronic interactions, unlike calculations from spectroscopic data. If the systematic errors affecting amplitudes determined by electron diffraction arise mainly from errors in scattering theory, internal agreement among diffraction amplitudes should in principle be a reliable indication of consistency among the true amplitudes. It turns out that, to within the relatively large stated uncertainties in the parameters, the electron diffraction amplitudes for all four molecules are identical, and in agreement with spectroscopic values. ${ }^{28}$
The above considerations give us confidence that $\mathrm{ReF}_{6}$ is a suitable choice for empirically characterizing deficiencies in the scattering experiment for the purpose of correcting the data for $\mathrm{ReF}_{7}$. An analysis of the electron diffraction intensities for the heptafluoride is presented in the following paper.

* This research was supported by a grant from the National Science Foundation. Based on a dissertation by E. J. Jacob in partial fulfillment of requirements for the degree of Doctor of Philosophy, University of Michigan, 1969. For a tabulation of experimental intensity data for $\mathrm{ReF}_{6}$ and $\mathrm{ReF}_{7}$, order Document NAPS-01042 from ASIS National Auxiliary Publications Service, c/o CCM Information Sciences, Inc., 22 W. 34th St., New York, N.Y. 10001 , remitting $\$ 1.00$ for microfiche or $\$ 3.00$ for photocopies.

$\dagger$ Author to whom correspondence concerning reprints should be addressed.

1 M. Kimura, V. Schomaker, D. W. Smith, and B. Weinstock, J. Chem. Phys. 48, 4001 (1968).

${ }^{2}$ H. M. Seip and R. Seip, Acta Chem. Scand. 20, 2698 (1966).

${ }^{3}$ H. M. Seip and R. Stölevik, Acta Chem. Scand. 20, 385 (1966).

${ }^{4}$ S. P. Arnesen and H. M. Seip, Acta Chem. Scand. 20, 2711 (1966).

${ }^{5}$ H. H. Claassen, E. O. Gasner, and H. Selig, J. Chem. Phys. $49,1803(1968)$ and references therein.

${ }^{6}$ W. J. Adams, doctoral dissertation, The University of Michigan, Ann Arbor, Mich., 1969.

${ }^{7}$ H. B. Thompson, Jr. and L. S. Bartell, Inorg. Chem. 7, 488 (1968) and references therein.

${ }^{8}$ W. Moffitt, G. L. Goodman, M. Fred, and B. Weinstock, Mol. Phys. 2, 109 (1959).

${ }^{9}$ B. Weinstock and G. L. Goodman, Advan. Chem. Phys. 9, 169 (1965); H. H. Claassen and H. Selig, Israel J. Chem. 7, 499 (1969).

${ }^{10}$ M. S. Child and A. C. Roach, J. Mol. Phys. 9, 281 (1965).

${ }^{11}$ L. S. Bartell, K. Kuchitsu, and R. J. de Neui, J. Chem. Phys. 35, 1211 (1961).

${ }_{12}^{12}$ T. L. Boates, Doctoral Dissertation, Iowa State University, Ames, Iowa, 1966.

${ }^{13}$ See asterisk footnote cited in byline.

${ }^{14}$ W. Heisenberg, Physik. Z. 32, 737 (1931); L. Bewilogua, ibid. 32, 740 (1931).

${ }^{15}$ H. L. Cox, Jr., Doctoral Dissertation, Indiana University, Bloomington, Ind., 1967.

${ }^{16}$ R. A. Bonham (unpublished calculations).

${ }^{17}$ Both the phase shifts and scattered amplitudes for rhenium had to be smoothed to remove oscillations of a well-defined period ( $\simeq 8 s$ units) and relatively large amplitude before they could be used in the present analysis.

${ }_{18} \mathrm{~J}$. A. Ibers and J. A. Hoerni, Acta Cryst. 7, 405 (1954).

19 Values for $s_{c}$ are given in the order (experimental, theoretical) for $40-\mathrm{kV}$ incident electrons. Although the Oslo electron difiraction group has published experimental determinations of $s_{c}$ for a number of molecules relevant to this study, they are generally for 35-kV electrons and thus not directly applicable to our work.

20 R. A. Bonham and T. Ukaji, J. Chem. Phys. 36, 72 (1962).

${ }^{21}$ It should be noted that the Bonham-Ukaji phase shifts have been shown to give too high an $s_{c}$ for such molecules as $\mathrm{XeF}_{6}$ and $\mathrm{IF}_{7}$. See Ref. 22 and H. B. Thompson. Jr., and L. S. Bartell, Trans. Am. Cryst. Assoc. 2, 190 (1966). Probably their behavior at large $s$ is inferior to that of Bonham's more recent values. This will have an effect on derived amplitudes of vibration for the rhenium fluorides. It is not clear why the more rigorous phase-shift calculations by Bonham should be at greater variance with experiment than the $\eta$ 's of Ibers and Hoerni, in respect to the value of $s_{c}$ anyway.

${ }_{22}$ R. M. Gavin, Jr. and L. S. Bartell, J. Chem. Phys. 48, 2460 (1968).

${ }^{23}$ See for example, L. S. Bartell and R. M. Gavin, Jr., J. Chem. Phys. 48, 2466 (1968); E. J. Jacob and L. S. Bartell, "Structure Analyses Combining Electron Diffraction and Microwave Data: Study of $\mathrm{XeOF}_{4}, " \mathrm{~J}$. Mol. Struct. (to be published), and Refs. $2-4$

${ }_{24}$ The improvement was on the order of $50 \%$ in $\sigma\left[I_{0}(s)\right]$ and $15 \%$ in $\sigma[f(r)]$ when uniform weighting of $I_{0}(s)$ was used, in 
contrast to the $10 \%$ improvements to both $\sigma\left[I_{0}(s)\right]$ and $\sigma[f(r)]$ when $s I_{0}(s)$ was weighted uniformly. The Oslo diffraction group have found similar increases in scale factors for nonbonded relative to bonded distances for $\mathrm{OsO}_{4}, \mathrm{WF}_{6}$, and $\mathrm{MoF}_{6}$ (see Refs. 2-4) but a decrease for $\mathrm{UF}_{6}$ and $\mathrm{TeF}_{6}$ (see Ref. 25).

${ }^{25}$ H. M. Seip, Acta Chem. Scand. 19, 1955 (1965); H. M. Seip and R. Stolevik, ibid. 20, 1535 (1966).
${ }^{26} \mathrm{H}$. Uehara, J. Chem. Phys. 45, 4536 (1966).

${ }^{27}$ S. J. Cyvin, Molecular Vibrations and Mean Square Amplitudes (Elsevier, Amsterdam, 1968), p. 240.

${ }^{28}$ Some disagreement over assignments of fundamental frequencies for $W_{6}$ (see Refs. 2 and 9 ) has led to a rather wide range of amplitudes calculated for this molecule.

\title{
Electron Diffraction Study of Rhenium Fluorides. II. Structure, Pseudorotation, and Anharmonic Coupling of Modes in $\mathrm{ReF}_{7}{ }^{*}$
}

\author{
E. JEAN JACOB AND L. S. BARTELL $\dagger$ \\ Department of Chemistry, University of Michigan, Ann Arbor, Michigan 48104
}

(Received 13 May 1970)

\begin{abstract}
Significant departures from $D_{5 h}$ symmetry are indicated by the gas-phase electron diffraction data for $\mathrm{ReF}_{7}$. Observed scattered intensities, corrected for heavy-atom effects as discussed in the preceding paper, are adequately represented by structures with static deformations of either $C_{2}$ or $C_{s}$ symmetry of the following character. A puckering of the ring of five fluorines (maximum out-of-plane displacement $\approx 9^{\circ}$ ) is accompanied by a movement of each axial fluorine from the reference axis of $\approx 8^{\circ}$ in the direction which provides maximum avoidance of fluorines on the rhenium coordination sphere. Equally compatible with the data is the much more reasonable dynamic pseudorotation model in which vibrational displacements carry the molecule from $C_{2}$ to $C_{3}$ to $C_{2}$ configurations. The tenfold character of the hindering potential and modest displacements preclude an appreciable barrier. A pronounced skewing of the envelope of axialequatorial, $F_{a} F_{e}$, distances reveals a coupling in phase of the $e_{1}^{\prime}$ axial bend to the $e_{2}^{\prime \prime}$ ring puckering vibration through the $S^{2}\left(e_{2}{ }^{\prime \prime}\right) S\left(e_{1}{ }^{\prime}\right)$ cubic term in the potential-energy function. The skewing, which moves the maximum of the $F_{a} F_{e}$ distribution peak inside the distribution center of gravity, results in an apparent "anharmonic shrinkage" of the nonbonded peak by an amount exceeding the well-known "BastiansenMorino" harmonic shrinkage. It is to be noted that the equatorial pseudorotation problem closely resembles that for cyclopentane in reduced mass, in displacement, and therefore, presumably, in frequency. The structure itself may be understood in terms of bond-bond repulsions thrusting the equatorial atoms out of plane; the out-of-plane displacement, in turn, induces an axial bend. The actual structure, however, exhibits too large an axial bend to conform to the simple repelling points-on-a-sphere model of Gillespie and too small a deviation from $D_{5 h}$ to correspond to the close packing of hard atoms around the rhenium.
\end{abstract}

\section{INTRODUCTION}

The molecule $\mathrm{ReF}_{7}$ offers a timely opportunity for assessing theories of directed valence for reasons outlined in the following paragraphs. A study of its vibrational spectrum led Claassen, Gasner, and Seligi,2 to conclude, tentatively, that the molecule has $D_{5 k}$ symmetry. Nevertheless, striking differences between the spectra of $\mathrm{IF}_{7}$, presumed by Claassen et al. to be $D_{5 h}$, and $\mathrm{ReF}_{7}$ left some doubts and raised questions about the internal motions involved. A more detailed characterization of the structure was desirable, and electron diffraction appeared to be the most direct method available.

Preliminary analyses of $\mathrm{IF}_{7},{ }^{3}$ and of $\mathrm{ReF}_{7}$, showed that the structures are appropriately described as pentagonal bipyramids. Nevertheless, small, persistent discrepancies between calculated $\left(D_{5 h}\right)$ and observed intensities (attributed initially but, incorrectly, for $\mathrm{IF}_{7}$ to sample impurities) ${ }^{3}$ disclosed that the mean structures of both molecules deviate appreciably from $D_{5 h}$ symmetry. Subsequently, it was found by Klemperer et al. ${ }^{4}$ that both molecules behave in an electrostatic focusing molecular-beam experiment as if they possess dipole moments, a behavior inconsistent with a simple $D_{5 h}$ structure. In the interpretation of available evidence some rather subtle points hinging on molecular dynamics are involved. It turns out to be helpful to consider these hand in hand with the structural theory we seek to shed light on.

In the hierarchy of structural theories the most fruitful place to begin when considering a molecule such as $\mathrm{ReF}_{7}$ is the Sidgwick-Powell-Gillespie-Nyholm valence-shell electron-pair-repulsion (VSEPR) theory. ${ }^{5-8}$ The link between this theory and less empirical valence theories is becoming clearer, and the VSEPR theory has been successful in many cases in accounting for structural $^{9}$ and vibrational ${ }^{10}$ properties in a simple and intuitively appealing manner. A useful variant of the VSEPR theory is one in which the strong mutual "Pauli repulsions" between occupied, localized molecular orbitals are represented by a repelling-points-ona-sphere (POS) model. The POS potential-energy terms for points $i$ and $j$ on the coordination sphere of the central atom are taken as $r_{i j}^{-n}$. Of all the simple compounds of form $\mathrm{MX}_{N}$ with $N$ valence shell pairs, the case of seven coordination is quite the most interesting. The reason for this is that the equilibrium 